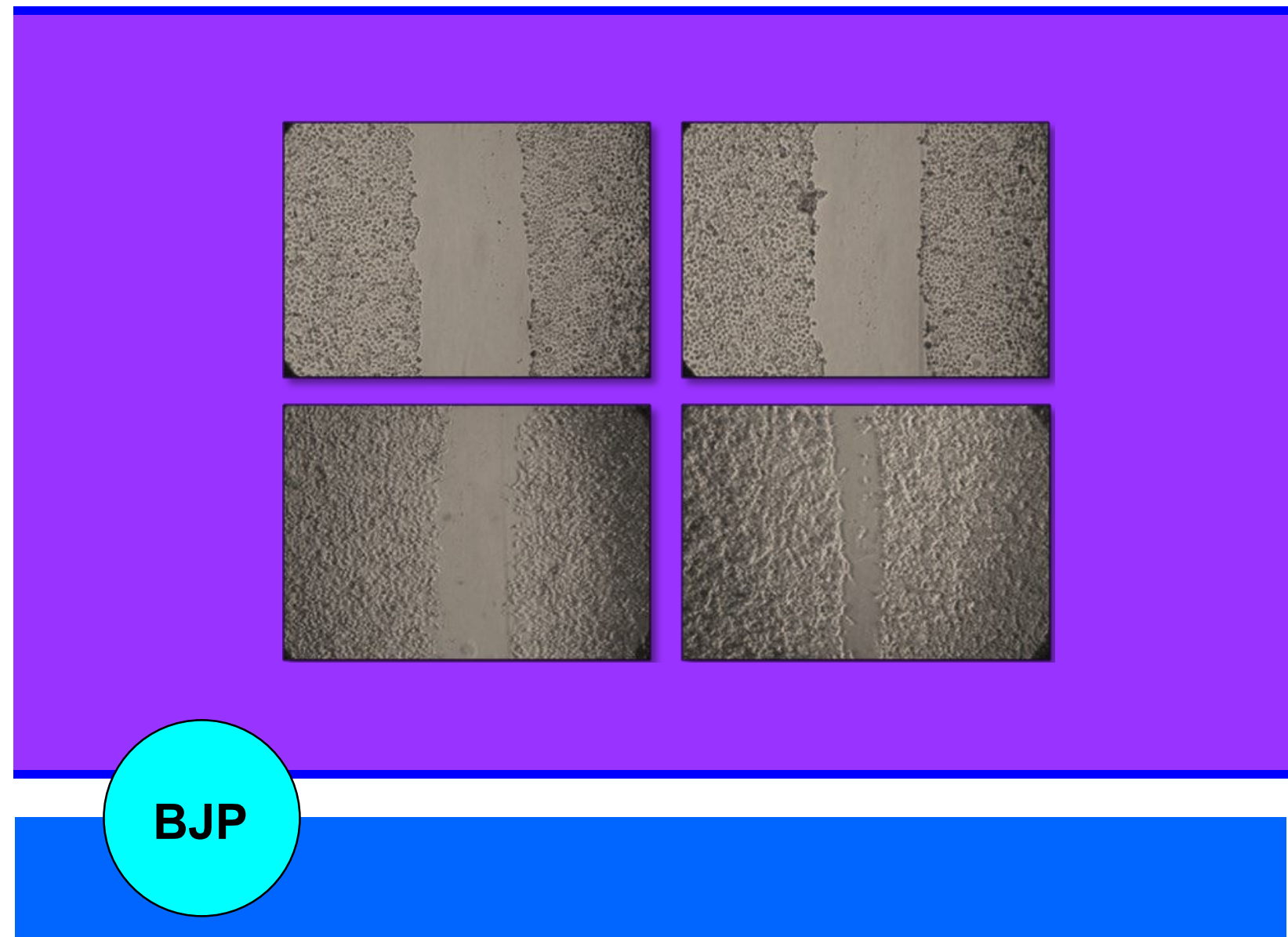

Bangladesh Journal of Pharmacology

Research Article

Kaempferol modulates the metastasis of human non-small cell lung cancer cells by inhibiting epithelialmesenchymal transition 


\title{
Kaempferol modulates the metastasis of human non-small cell lung cancer cells by inhibiting epithelial-mesenchymal transition
}

\author{
Meng Hang, Fan Zhao, Shu-Bo Chen, Qing Sun and Chun-Xiao Zhang \\ Department of Oncology, Jiangsu Province Wuxi No. 2 People's Hospital, Wuxi 214002, China.
}

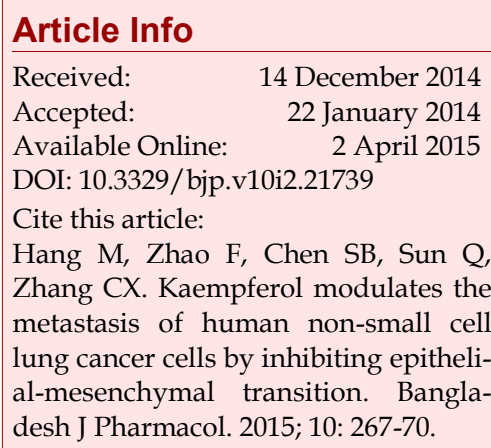

Article Info

Available Online:

Cite this article:

Hang M, Zhao F, Chen SB, Sun Q, Zhang CX. Kaempferol modulates the lung cancer cells by inhibiting epithelidesh J Pharmacol. 2015; 10: 267-70.

\begin{abstract}
The present study was done to determine whether kaempferol, a natural polyphenol of the flavonoid family, affects Epithelial-Mesenchymal Transition (EMT) in non-small cell lung cancer cells. Kaempferol not only inhibited cancer cell proliferation and migration in a dose-dependent manner but also modulated the expression of EMT-related proteins E-cadherin and vimentin which are indispensable to cellular motility, invasiveness and metastasis. These results indicate that kaempferol suppresses non-small cell lung cancer migration by modulating the expression of EMT proteins. Therefore, kaempferol may be useful as a potential anticancer agent for non-small cell lung cancer.
\end{abstract}

\section{Introduction}

Lung cancer is the most commonly diagnosed cancer type worldwide and a leading cause of cancer-related deaths. It can be categorized into two sub-types: nonsmall cell lung cancer (Koh et al., 2012) and small cell lung cancer. Despite many efforts to improve lung cancer outcome, long-term survival has not improved significantly over the last 20 years, with a 5-year cumulative survival rate that remains very dismal at only $15 \%$ (Ghosal et al., 2009). Current standard therapies limited to chemotherapy and radiotherapy or both rarely cure this disease, thus accentuating the need for more effective and alternate therapeutic strategies.

Metastasis is a complex, multistep process and involves the invasion of cells from primary tumors into the circulation, migration to distant organs and finally infiltration into tissues referred to as secondary metastatic sites (Gupta and Massagué, 2006). Epithelial-mesenchymal transition (EMT), a developmental program plays an important role in this process and involves downregulation of epithelial markers like E-cadherin and upregulation of mesenchymal markers like vimentin and fibronectin (Borthwick et al., 2009; Kalluri and Neilson, 2003). As a result, the epithelial cells acquire fibroblast- like properties, thus losing their defined cell-cell and cell-extracellular matrix contacts (Thiery et al., 2010). Thus, the EMT becomes a target to prevent tumor progression.

Flavonoids are polyphenolic natural compounds present in a wide variety of fruits and vegetables (Bosetti et al., 2007) and in recent years their anti-tumor activities have been widely studied and recognized (Gonzalez and Riboli, 2006). Kaempferol (3,4',5,7-tetrahydroxyflavone), is a natural polyphenol of the flavonoid family and exhibits various biological properties including anti -tumor activities. It induces apoptosis and cell cycle arrest in various cancer cell lines, including lung cancer cells (Nguyen et al., 2003), breast cancer cells (Kang et al., 2010), colon cancer cells (Li et al., 2009), besides inhibiting the migration and invasiveness of glioma cells (Shen et al., 2006). The effect of kaempferol on the growth and invasiveness of lung cancer is not yet determined and the mechanism involved needs to be defined. The present study examined the effects of kaempferol on the metastasis and invasion of A549 non-small cell lung cancer cells. It was found that kaempferol markedly inhibited cell proliferation besides overcoming EMT and cell migration. 


\section{Materials and Methods}

Cell culture and kaempferol treatment: A549 cells were maintained in DMEM (Hyclone) supplemented with $10 \%$ fetal bovine serum (Gibco), 100U/mL penicillin and $100 \mathrm{mg} / \mathrm{mL}$ streptomycin. The cells were grown under standard culture conditions at $37^{\circ} \mathrm{C}$ under $5 \%$ $\mathrm{CO}_{2}$ in a humidified incubator. The medium was changed every two days or until the cells became confluent and then used for further experimentation. Stock solution of $100 \mathrm{mM}$ kaempferol (Sigma-Aldrich) was prepared in DMEM and diluted to different concentrations.

Cell proliferation assay: The effect of kaempferol on A549 cells was evaluated using MTT cell proliferation assay. The cell suspension containing about 10,000 cells was seeded into each well of a 96 well plate. The cells were allowed to grow for 24 hours after which different concentrations of kaempferol were added and incubited for 24 hours. MTT (Sigma-Aldrich) solution was added to the cells at a concentration of $0.1 \mathrm{mg} / \mathrm{mL}$ (dissolved in PBS) followed by incubation for 4 hours at $37^{\circ} \mathrm{C}$ in dark. The supernatant was removed and $100 \mu \mathrm{L}$ of DMSO was added to dissolve the formazan crystals. The optical density was measured at $490 \mathrm{~nm}$ using a plate reader (Bio-Rad, Hercules, CA). The results were presented as the percentages relative to the controls. The percentage proliferation inhibition rate was calculated as $=(1-$ OD sample $/$ OD control $) \times 100 \%$.

Western blotting: Cells were lysed in ice cold RIPA buffer (50 mM Tris- $\mathrm{HCl}$ (pH 7.6), $150 \mathrm{mM} \mathrm{NaCl}, 1 \mathrm{mM}$ EDTA, 1\% NP-40, 0.5\%Na-deoxycholate) supplemented with protease inhibitors. The protein concentration was determined by BCA method (Thermo Scientific Pierce). Twenty five micrograms of each protein sample were separated on a $10 \%$ polyacrylamide gel and electro-transferred to PVDF membrane. The membrane was then blocked with 5\% non-fat dried milk in PBS at room temperature for 2 hours, followed by incubation with primary antibodies against E-cadherin (Santa Cruz Biotechnology), vimentin (Santa Cruz Biotechnology) and $\beta$-actin (Sigma) at $4^{\circ} \mathrm{C}$ overnight. After washing, the blots were hybridized with secondary goat antirabbit antibodies (Sigma) for 1 hour at room temperature and developed using an enhanced chemiluminescence detection system (Amersham).

Wound healing assay: A549 cells were plated in $30 \mathrm{~cm}$ cell culture dishes and grown up to $80 \%$ confluence. The media was then removed and the cells scratched with $100 \mu \mathrm{L}$ pipette tip. Cells were then washed with PBS to remove detached cells, photographed $(t=0$ hour $)$ and treated with kaempferol for 24 hours. Then the wounds were observed and photographed ( $\mathrm{t}=48$ hours).

\section{Results}

The antiproliferative activity of kaempferol was tested by MTT assay against human non-small lung cancer cell line, A549. A549 cells showed a significant growth inhibition at all the tested concentrations of kaempferol. The effect of different concentrations on the proliferation of A549 cells was seen to be dose-dependent. The results demonstrated that percentage of growth inhibition increased with increasing concentration of kaempferol from 10 to $140 \mu \mathrm{M}$ with an $\mathrm{IC}_{50}$ value of $72 \mu \mathrm{M}$ after 24 hours of incubation (Figure 1). Kaempferol exhibited maximum anti-proliferative potential at a concentration of $140 \mu \mathrm{M}$ and lowest at $10 \mu \mathrm{M}$. Only such concentrations (10, 20, 40 and $60 \mu \mathrm{M})$ which exhibited lesser influence on the viability of A549 cells were used in all subsequent experiments

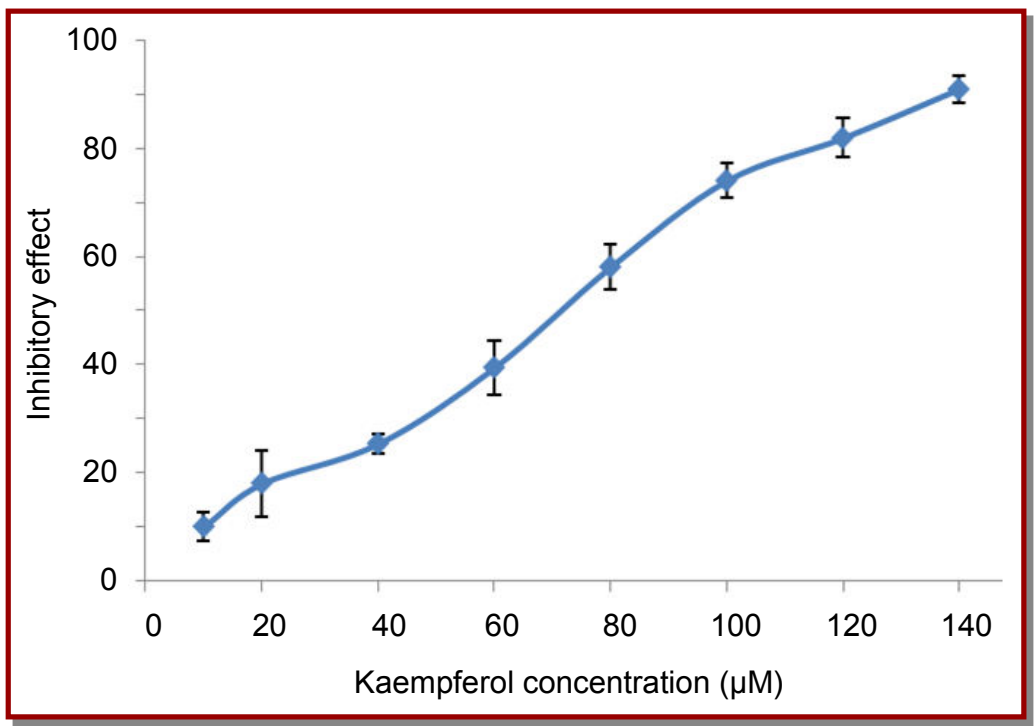

Figure 1: Inhibitory effects of kaempferol on the proliferation of non-small cell lung cancer cells. A549 cells were treated with increasing concentrations of kaempferol for 24 hours 


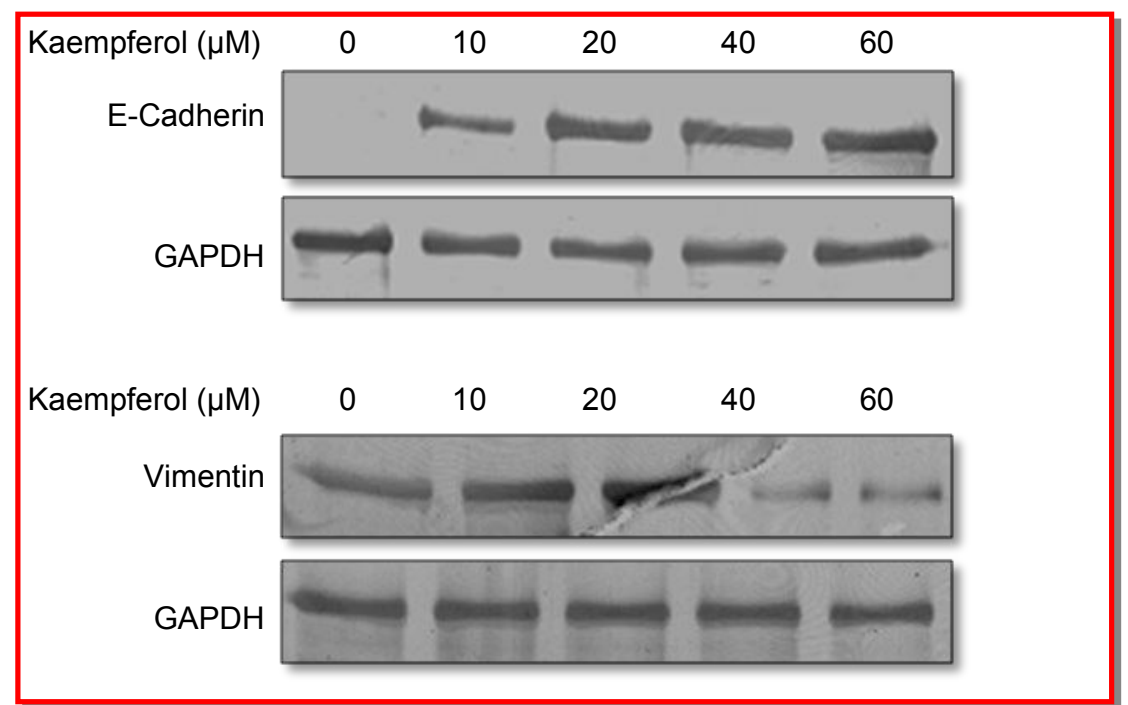

Figure 2: Effects of kaempferol on the expression of EMT markers in non-small cell lung cancer cells. A549 cells were treated with increasing concentrations of kaempferol for 24 hours and the protein expression levels of E-cadherin and vimentin were detected

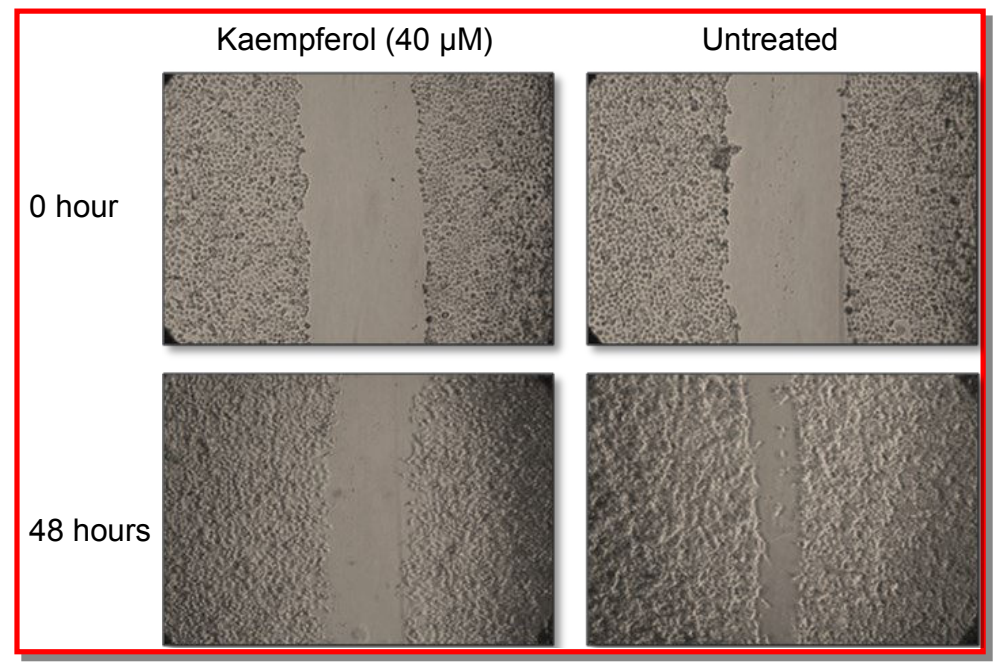

Figure 3: Effects of kaempferol on the migration of non-small cell lung cancer cells. A549 cells were either treated with $40 \mu \mathrm{M}$ of kaempferol or left untreated (control) for 24 hours and the wound healing was observed after 48 hours

EMT imparts migratory abilities which in turn enhance the invasive capabilities of cancer cells. Once switched on, this process leads to unfavorable prognosis in many different types of cancers.

We examined the effect of kaempferol on the expression of EMT-related proteins E-cadherin and vimentin after incubation of cells with different concentrations (10, 20, 40 and $60 \mu \mathrm{M}$ ) for 24 hours. Western blotting showed that kaempferol treatment in a dose-dependent manner enhanced the expression of E-cadherin with concomitant decrease in expression of vimentin, thereby limiting EMT (Figure 2).

Since the concentration of $40 \mu \mathrm{M}$ was very effective in modulating the expression of E-cadherin and vimentin, we therefore tried to evaluate whether kaempferol at similar concentration could also affect the metastatic behaviour of A549 cells. A549 cells were grown to subconfluency, wounded and then treated with $40 \mu \mathrm{M}$ kaempferol for 24 hours. Kaempferol significantly overcame migratory abilities of A549 cells in comparison to control (untreated) as was reflected by attenuated wound healing (Figure 3).

\section{Discussion}

There is considerable epidemiological evidence which shows that cancer risk decreases with higher intake of vegetables, fruits, and grains (Witte et al., 1996). Flavonoids, commonly found in fruits and vegetables have been widely recognized for their remarkable anti- 
carcinogenic properties (Hoensch et al., 2005). Kaempferol, is a member of the flavonoid family and exhibits potential anti-tumor activities. Previous studies demons -trated that kaempferol inhibits growth and proliferation of cancer cells by various mechanisms including apoptosis (Sharma et al., 2007) cell cycle arrest (Cho et al., 2013) and inhibition of tyrosine phosphorylation (Lee et al., 1998). However, studies focusing on the potential of kaempferol in overcoming invasiveness of cancer cells particularly lung cancer have rarely been done. Therefore, the present study was performed to evaluate the potential of kaempferol in overcoming the invasiveness of human non-small cell lung cancer cells.

In this study, kaempferol was potent in inhibiting the proliferation of A549 cells. This effect was dose dependent with an $\mathrm{IC}_{50}$ value of $72 \mu \mathrm{M}$. To assess the efficacy of kaempferol in the lung cancer progression, A549 cells were treated with different concentrations of kaempferol for 24 hours and the expression levels of EMT markers E-cadherin and vimentin monitored by western blotting. Kaemferol increased the expression of the epithelial marker, E-cadherin in A549 cells in a dose -dependent manner. In addition, there was downregulation of mesenchymal protein marker, vimentin by kaempferol treatment. There was inhibition of A549 cell migration when treated with the most efficacious concentration of kaempferol, as evaluated by the wound-healing assay. The finding suggests the role of kaempferol in impeding the EMT in A549 cells and provides an evidence for the use of kaempferol against non-small cell lung cancer progression.

\section{References}

Borthwick LA, Parker SM, Brougham KA et al. Epithelial to Mesenchymal Transition (EMT) and airway remodelling after human lung transplantation. Thorax 2009; 64: 770-77.

Bosetti C, Rossi M, McLaughlin JK, Negri E, Talamini R, Lagiou P, Montella M, Ramazzotti V, Franceschi S, LaVecchia C. Flavonoids and the risk of renal cell carcinoma. Cancer Epidemiol Biomarkers Prev. 2007; 16: 98101.

Cho HJ, Han J, Park Y. Kaempferol induces cell cycle arrest in HT-29 human colon cancer cells. J Cancer Prev. 2013; 18: 257 $-63$.

Ghosal R, Kloer P, Lewis KE. A review of novel biological tools used in screening for the early detection of lung cancer. Postgrad Med J. 2009; 85: 358-63.
Gonzalez CA, Riboli E. Diet and cancer prevention: Where we are, where we are going. Nutr Cancer. 2006; 56: 225-31.

Gupta GP, Massagué J. Cancer metastasis: Building a framework. Cell 2006; 127: 679-95.

Hoensch HP, Kirch W. Potential role of flavonoids in the prevention of intestinal neoplasia: A review of their mode of action and their clinical perspectives. Int J Gastrointest Cancer. 2005; 35: 187-95.

Kalluri R, Neilson EG. Epithelial-mesenchymal transition and its implications for fibrosis. J Clin Invest. 2003; 112: 1776-84.

Kang JW, Kim JH, Song K, Kim SH, Yoon JH, Kim KS. Kaempferol and quercetin, components of Ginkgo biloba extract (EGb 761), induce caspase-3-dependent apoptosis in oral cavity cancer cells. Phytother Res. 2010; 24(Suppl 1): S77 -82 .

Koh PK, Faivre-Finn C, Blackhall FH, De Ruysscher D. Targeted agents in non-small cell lung cancer (NSCLC): Clinical developments and rationale for the combination with thoracic radiotherapy. Cancer Treat Rev. 2012; 38: 62640.

Lee SC, Kuan CY, Yang CC, Yang SD. Bioflavonoids commonly and potently induce tyrosine dephoshorylation/inactivation of oncogenic proline-directed protein kinase FA in human prostate cancer cells. Anticancer Res. 1998; 18: 111721.

Li W, Du B, Wang T, Wang S, Zhang J. Kaempferol induces apoptosis in human HCT116 colon cancer cells via the ataxia -telangiectasia mutated-p53 pathway with the involvement of p53 up-regulated modulator of apoptosis. Chem Biol Interact. 2009; 177: 121-27.

Nguyen TT, Tran E, Ong CK, Lee SK, Do PT, Huynh TT, et al. Kaempferol-induced growth inhibition and apoptosis in A549 lung cancer cells is mediated by activation of MEKMAPK. J Cell Physiol. 2003; 197: 110-21.

Shen SC, Lin CW, Lee HM, Chien LL, Chen YC. Lipopolysaccharide plus 12-O tetradecanoylphorbol 13-acetate induction of migration and invasion of glioma cells in vitro and in vivo: Differential inhibitory effects of flavonoids. Neuroscience 2006; 140: 477-89.

Thiery JP, Chua K, Sim WJ, Huang R. Epithelial to mesenchymal transition during development in fibriossis and in the progression of carcinoma. Bull Cancer. 2010; 97: 1285-95.

Sharma V, Joseph C, Ghosh S, Agarwal A, Mishra MK, Sen E. Kaempferol induces apoptosis in glioblastoma cells through oxidative stress. Mol Cancer Ther. 2007; 6: 2544-53.

Witte JS, Longnecker MP, Bird CL, Lee ER, Frankl HD, Haile RW. Relation of vegetable, fruit, and grain consumption to colorectal adenomatous polyps. Am J Epidemiol. 1996; 144: 1015-25. 